

\section{Hybrid Entrepreneurship as a Tool for Poverty Reduction in Nigeria: A Review of Literature with Qualitative Research and Content Analysis}

\author{
Olajide Olubayo Thomas \\ Olajide Idowu Okunbanjo
}

\section{Introduction}

Poverty has become a major concern for world leaders, governments, international bodies, nongovernmental bodies, individuals as well as researchers. As a matter of fact, poverty is a great challenge in the globe. As Aristotle states, "Poverty is the parent of revolution and crime" (Aristotle, 3843-22BC). The concern has forced the local and international bodies to focus on methods to eliminate and to reduce poverty rate in the world, especially in developing economies.

Ezeanyeji, Imoagwu and Ejefobihi (2019) mention that when there is no development in the entrepreneurial or business activities, the daily income of individuals could be stagnant or reduced. Therefore, there is need for the development of business activities in order for poverty to be decreased in any economy. One of the entrepreneurial or business activities identified is hybrid entrepreneurship (Kurczewska, Mackiewicz, Doryń, \& Wawrzyniak, 2020; Luc, Chirita, Delvaux \& Kepnou, 2018; Dzomonda \& Fatoki, 2018; Solesvik, 2017; Raffiee \& Feng, 2014). Kurczewska et. al. (2020) posit that, hybrid entrepreneurship brings a new employment phenomenon into entrepreneurial practices and theory. The state of developing economies like Nigeria has forced the people to think and rethink on a source of income to achieve their personal and family goals. Hybrid entrepreneurship is more of a creative and supportive entry mode into full entrepreneurship (Dzomonda \& Fatoki, 2018; Xi, Block, Lasch, Robert \& Thurik, 2017; Raffiee \& Feng, 2014). As noted by Viljamaa and Varamäki (2015), hybrid entrepreneurship serves as a vantage platform for learning which allows individuals to develop relevant entrepreneurial skills for entrepreneurial venture (Solesvik, 2017) and establish the market demand for their offering (Raffiee \& Feng, 2014).

In Africa at large, poverty has been viewed as part of the continental characteristics due to the high level of unemployment and high rate of inflation. Nigeria as a developing economy in Africa is faced with a series of challenges that have led to poor living conditions which has resulted in poverty. Umaru and Ibrahim (2019) point that, poverty level is identified when the daily income in an economy is less than $\$ 1.25$ or exactly $\$ 1.25$ and contextual observation indicates that most daily income in Nigeria is not up to $\$ 1.25$. This signifies the evidence of poverty in Nigerian economy which requires vigorous efforts from the government to curb this menace.
Nigeria is rated second in the list of poorest countries all over the world in recent times. Among the reasons accounted for this high level of poverty is the orientation towards paid employment. Most Nigerians that finish secondary school or university are looking for paid employment. This was going on well after the country's independence in 1960 till say 1980s. But the situation changes as the jobs are no longer able to sustain the population depending on the income (salary). The population has grown geometrically. Besides, the jobs are no longer there. The few who are still having the jobs are now considering how to sustain themselves, thus, giving birth to hybrid entrepreneurship. Necessity of the present situation (economic situation) could be attributed to the development of hybrid entrepreneurship in Nigeria even though this is not the case in many other climes. Poverty and degree of need is making many that even have jobs to think out of the box on how to survive, hence hybrid entrepreneurship.

According to Olajide (2018), several concerted efforts have been made by the federal government of Nigeria to reduce the level of poverty through entrepreneurship programs. Among the programs introduced include Small and Medium-Size Enterprise Development Association of Nigeria (SMEDAN), The Industrial Development Coordinating Centre (IDDC), Family Economic Advancement Programme (FEAP), Youth Enterprise with Innovation in Nigeria (YouWIN), Industrial Training Institute (ITI), Young Entrepreneur Scheme (YES), Trader Moni among others. Yet, there is still a strong existence of high poverty level in Nigeria and poverty still dominates many parts of the globe (Ezeanyeji et al 2019; Umaru \& Ibrahim, 2019; Hussain \& Noma, 2019; Alvarez \& Barney, 2014). Thus, the question of what other means can be done for poverty to be reduced in Nigeria arises. In the opinion of Bogenhold (2018), hybrid entrepreneurship could make an individual entrepreneurial billionaire. Therefore, it could be postulated that hybrid entrepreneurship could aid in reducing poverty level. Thus, it is important to see how hybrid entrepreneurship has been used as a tool for poverty reduction in Nigeria.

\section{Hybrid Entrepreneurship}

Luc et. al. (2018) see hybrid entrepreneurship as the process that involves combination of self-employment and wage labor. It is a situation whereby an individual combines both paid job and entrepreneurial venture together. It implies that an individual works and still engages in self-employment activities. Hammarström and Nylén (2014) see hybrid entrepreneurship as the means which a person tries out a business idea and business entrepreneurial skills while still maintains wage-work in order to alleviate personal financial risk. According to Dzomonda and Fatoki (2018), hybrid entrepreneurship is a situation where individuals integrate their time in both self and wage employment. In other words, the individual has a business of his own while he/she still works as an employee. Thus, hybrid entrepreneurship could be the mixture of an individual being an employee as well as employer in different organizations. Hammarström and Nylén (2014) further see hybrid entrepreneurship as method for minimization of personal risk, which involves entrepreneurial venture and paid employment. 
Hybrid entrepreneurship has been confused with part-time entrepreneurship. Some studies state that hybrid entrepreneurship is the same with part-time entrepreneurship (Luc et al 2018; Schulz, Urbig, \& Procher, 2016; Petrova, 2012) while some studies state that hybrid entrepreneurship is different from part-time entrepreneurship (Dzomonda \& Fatoki, 2018; Nordstrom, 2015; Hammarström \& Nylén, 2014; Viljamaa \& Varamäki, 2014; Folta, Delmar, \& Wennberg, 2010; Petrova, 2010; Kim, Aldrich \& Keister, 2006). Nordstrom (2015) is of the view that part time entrepreneurship covers a series of alternatives compared to hybrid entrepreneurship. Part-time entrepreneurship could make an individual to mix unemployment and entrepreneurship at a go or it could be having a series of portfolio businesses (Nordstrom, 2015; Petrova, 2012). Part-time entrepreneur is recognized by having a normal wage job while balancing the work with personal business at the same time (Petrova, 2010). An individual can only engage in part-time entrepreneurship in as much as the individual is not under a paid or wage job while practicing entrepreneurship. Specifically, the combination of paid employment and self-employment is known to be hybrid entrepreneurship (Kurczewska et al., 2020; Dzomonda \& Fatoki, 2018)

Hammarström and Nylén (2014) narrate that, hybrid entrepreneurship is aimed at attempting business or entrepreneurial idea while still under employment contract with an organization. This is a strategy in which an individual tries his/her entrepreneurial abilities and the opportunities of his/her ideas in order to minimize personal financial risk while still keeping a wage-work. Hybrid entrepreneurship serves as an understanding of the practice of entrepreneurship without worrying about income or profit. In other words, it is preentrepreneurship practice. Therefore, a hybrid entrepreneur may have the intention of becoming a full entrepreneur (Kurczewska et al., 2020). Part-time entrepreneurship has the sole aim to complement the current income of an individual. A part-time entrepreneur does not have the aim of becoming a full entrepreneur unlike a hybrid entrepreneur. A part-time entrepreneur sticks to the wage job while being able to meet his/her other financial obligations with a side venture (Hammarström \& Nylén, 2014). Basically, hybrid entrepreneurship is associated with the early stages of entrepreneurial venture development. It is worth noting that, establishing a part-time business is not necessarily indicative of a desire to enter full-time entrepreneurship (Viljamaa, Varamäki, \& Joensuu-Salo, 2017).

Hybrid entrepreneurship could be established based on passion motive, changes in employment market, job insecurity or uncertainty, creative challenges, contract job status, non-standard working arrangements, government policy, desire for autonomy or independence, a need for social recognition and additional income among others (Dzomonda \& Fatoki, 2018; Luc et al., 2018; Clark, 2018; Solesvik, 2017; Popiel, 2017; Schulz et al., 2016; Kelley, Singer, \& Herrington, 2016; Kuhn, 2016; Florida, 2014).

\section{Poverty Reduction}

Poverty is seen as a condition of low-income or low consumption (Ezeanyeji et al., 2019). Poverty is the inability of individuals in an economy to attain a minimum standard of living (World Bank Report, 1990).
Ibotoye, Atoyebi and Sufian (2015) refer poverty as the absence of command over basic consumption needs such as insufficient food, clothing and/or shelter while Olayemi (1995) refers to the poor as those having no access to the basic necessities of life such as food, clothes and economic obligation. They lack skillful employment, have few, if any economic assets and sometime lack self-esteem. World Bank (2011) sees poverty as an economic situation where people lack plenty income to obtain certain minimal levels of health services, food, housing, clothing and education which are necessities for the standard of living. Therefore, poverty is the incapacity of people in an economy to meet basic needs of life.

Ikechukwu, Onyi and Edeh (2017) divide poverty into three categories: Absolute poverty, relative poverty and subjective poverty. Absolute poverty is a situation where an individual is constrained with limited financial resources and he is unable to meet his/her basic needs of life such as food, clothes, shelter and health. Relative Poverty is a situation where an individual's income is less than the average income of the population in the society being considered. Subjective poverty is based on the individual perception about an individual's standard of living.

Poverty reduction is the alleviation of level of poverty in an economy. Ezeanyeji et. al. (2018) identify Agricultural Development Programmes (ADPs), Directorate of Food, Roads and Rural Infrastructure (DFRRI), National Agricultural Land Development Authority (NALDA), Better Life Programme (BLP), National Directorate of Employment (NDE), People' Bank of Nigeria (PBN), Agricultural Credit Guarantee Scheme, River Basin Development Authorities, Community Bank and Interventions from International Development Agencies among others as policies or strategies employed since the inception of Nigeria to reduce poverty.

\section{Lazear Theory of Entrepreneurship}

Lazear Theory of Entrepreneurship is also known as jack-of-all-trades theory. It is propounded by Edward Lazear in 2002. Astebro and Thompson (2011), BackesGellner and Moog (2013), Hartog, Van Praag and Van Der Sluis (2010), Stuetzeret, Obschonka, Davidsson and Schmitt-Rodermund (2013), Wagner (2003) and Wagner (2006) are among the supporters of Lazear Theory of Entrepreneurship.

Lazear Theory of Entrepreneurship indicates that, entrepreneurs should be jacks-of-all-trades in order to have broad and diverse knowledge, skills and professional experience in different aspects of business (Kurczewska \& Mackiewicz, 2020). The theory explains an individual selection into entrepreneurship (Hsieh, Parker and van Praag, 2017), which signifies alternative perception to the risk aversion theory. The theory considers that individuals with a balanced but diversified combination of skills and knowledge, collected from diverse sources and domains, are more likely to become entrepreneurs, therefore capturing hybrid entrepreneurship in theory and in practice.

Despite the relevance of Lazear Theory of Entrepreneurship to the practice and concept of hybrid entrepreneurship, Kurczewska and Mackiewicz (2020) state that this theory fails to capture how entrepreneurs as jacks-of-all-trades become successful. Thus, 
entrepreneurial performance is not captured in the theory. In the same vein, it is also pointed that self-efficacy is not included in Lazear Theory of Entrepreneurship as a factor that predicts individual entry into entrepreneurship. Lazear did not include it in his set of factors explaining entrepreneurial career (Tegtmeier, Kurczewska \& Halberstadt, 2016).

\section{Conceptual Framework}

The conceptual framework of research is indicated in Figure 1 below. As it is observed, three factors which are paid employment, hybrid entrepreneurship and selfemployment affect poverty reduction.

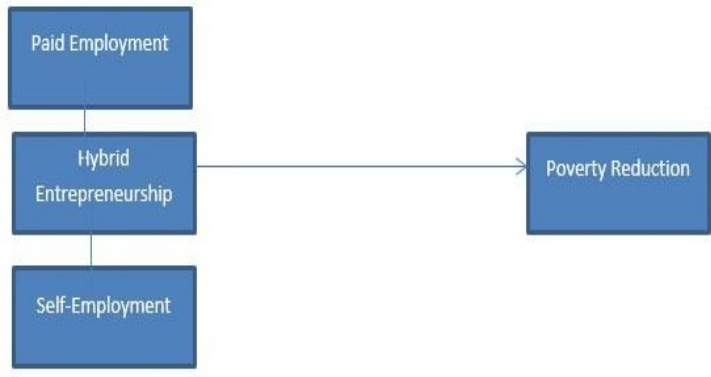

Figure 1. Conceptual Framework of Research

Source: Researchers' Conceptual Framework, 2021

\section{Research Methodology}

The study adopts qualitative research approach and employs exploratory research design. The paper makes use of secondary data and the data are retrieved via review of the past studies on hybrid entrepreneurship. The study employs a critical review on past studies on the subject matter so as to identify the relationship that exists between hybrid entrepreneurship and poverty reduction. Content analysis is used to analyze the observations in the studies reviewed.

Review of Studies on Hybrid Entrepreneurship and Poverty Reduction

The review of studies on hybrid entrepreneurship and poverty reduction via qualitative research and content analysis are listed below in Table 1:

Table 1: Studies on Hybrid Entrepreneurship and Poverty Reduction

\begin{tabular}{|c|c|c|c|c|c|}
\hline $\begin{array}{l}\mathbf{S} / \\
\mathbf{N}\end{array}$ & $\begin{array}{l}\text { Author } \\
\text { Name } \\
\text { and } \\
\text { Year }\end{array}$ & Title & $\begin{array}{l}\text { Methodo } \\
\text { logy }\end{array}$ & Findings & Gap \\
\hline 1 & $\begin{array}{l}\text { Demir, } \\
\text { Werner } \\
\text { and } \\
\text { Kraus } \\
(2020)\end{array}$ & $\begin{array}{l}\text { Hybrid } \\
\text { entrepreneur } \\
\text { ship: A } \\
\text { systematic } \\
\text { literature } \\
\text { review }\end{array}$ & $\begin{array}{l}\text { Qual. } \\
\text { research } \\
\text { approach, } \\
\text { syste } \\
\text { matic } \\
\text { literature } \\
\text { review } \\
\text { and } \\
\text { content } \\
\text { analysis }\end{array}$ & $\begin{array}{l}\text { The study } \\
\text { concludes } \\
\text { that } \\
\text { there is a } \\
\text { missing } \\
\text { consensus } \\
\text { in applied } \\
\text { terminolog } \\
\text { y, criteria } \\
\text { and } \\
\text { conception } \\
\text { of hybrid }\end{array}$ & $\begin{array}{l}\text { Poverty } \\
\text { reduc } \\
\text { tion is } \\
\text { not } \\
\text { capture } \\
\mathrm{d} \text { in the } \\
\text { study } \\
\text { and it is } \\
\text { not a } \\
\text { paper } \\
\text { authore } \\
\text { d by }\end{array}$ \\
\hline
\end{tabular}

\begin{tabular}{|c|c|c|c|c|c|}
\hline & & & & $\begin{array}{l}\text { entre. in } \\
\text { theory and } \\
\text { practice }\end{array}$ & $\begin{array}{l}\text { Nigeria } \\
\mathrm{n} \\
\text { research } \\
\text { ers }\end{array}$ \\
\hline 2 & $\begin{array}{l}\text { Aladeje } \\
\text { bi } \\
(2020)\end{array}$ & $\begin{array}{l}\text { Hybrid } \\
\text { Entrepreneu } \\
\text { rship: } \\
\text { Combining } \\
\text { Employmen } \\
t \text { and Self } \\
\text { Employmen } \\
t \text { in Nigeria }\end{array}$ & $\begin{array}{l}\text { Quant. } \\
\text { research } \\
\text { approach, } \\
\text { purposive } \\
\text { sampling } \\
\text { and } \\
\text { descrip } \\
\text { tive } \\
\text { statistics }\end{array}$ & $\begin{array}{l}\text { Most of the } \\
\text { hybrid } \\
\text { business } \\
\text { owners } \\
\text { were } \\
\text { employees } \\
\text { in the } \\
\text { public } \\
\text { sector and } \\
\text { they are } \\
\text { approxi } \\
\text { mately } 31- \\
50 \text { years } \\
\text { old. Also, } \\
\text { autonomy } \\
\text { and desire } \\
\text { for extra } \\
\text { income are } \\
\text { the main } \\
\text { reasons for } \\
\text { running } \\
\text { hybrid } \\
\text { entrepre } \\
\text { neurship }\end{array}$ & $\begin{array}{l}\text { Poverty } \\
\text { reduc } \\
\text { tion is } \\
\text { not } \\
\text { conside } \\
\text { red in } \\
\text { the } \\
\text { study, } \\
\text { althoug } \\
\text { h this } \\
\text { research } \\
\text { was } \\
\text { conduc } \\
\text { ted in } \\
\text { Nigeria }\end{array}$ \\
\hline 3 & $\begin{array}{l}\text { Ferreira, } \\
(2020)\end{array}$ & $\begin{array}{l}\text { Experiential } \\
\text { learning } \\
\text { theory and } \\
\text { hybrid } \\
\text { entrepre } \\
\text { neurship: } \\
\text { Factors } \\
\text { influencing } \\
\text { the } \\
\text { transition to } \\
\text { full-time } \\
\text { entre } \\
\text { preneurship }\end{array}$ & $\begin{array}{l}\text { Sec. data } \\
\text { analysis } \\
\text { and } \\
\text { thematic } \\
\text { analysis }\end{array}$ & $\begin{array}{l}\text { It indicated } \\
\text { hybrid } \\
\text { entrep } \\
\text { reneurship } \\
\text { as an } \\
\text { effective } \\
\text { learning } \\
\text { ground and } \\
\text { path toward } \\
\text { full-time } \\
\text { entre } \\
\text { preneurship }\end{array}$ & $\begin{array}{l}\text { The } \\
\text { study is } \\
\text { not } \\
\text { empiric } \\
\text { ally } \\
\text { conduc } \\
\text { ted and } \\
\text { poverty } \\
\text { reduc } \\
\text { tion is } \\
\text { not } \\
\text { capture } \\
\text { d. More } \\
\text { over, } \\
\text { the } \\
\text { study is } \\
\text { not } \\
\text { conduc } \\
\text { ted in } \\
\text { Nigeria }\end{array}$ \\
\hline 4 & $\begin{array}{l}\text { Kurcze } \\
\text { wska et. } \\
\text { al. } \\
(2020)\end{array}$ & $\begin{array}{l}\text { Peculiarity } \\
\text { Of Hybrid } \\
\text { Entre } \\
\text { preneurs- } \\
\text { Revisiting } \\
\text { Lazear's } \\
\text { Theory of } \\
\text { Entre } \\
\text { preneurship }\end{array}$ & $\begin{array}{l}\text { Survey } \\
\text { research } \\
\text { design, } \\
\text { Primary } \\
\text { data and } \\
\text { Logistic } \\
\text { regressio } \\
\text { n }\end{array}$ & $\begin{array}{l}\text { Professiona } \\
1 \text { and } \\
\text { managerial } \\
\text { experiences } \\
\text { enhance } \\
\text { hybrid } \\
\text { entre } \\
\text { preneurship } \\
\text { than } \\
\text { education } \\
\text { experience }\end{array}$ & $\begin{array}{l}\text { The } \\
\text { study } \\
\text { does not } \\
\text { focus } \\
\text { on } \\
\text { poverty } \\
\text { level } \\
\text { reduc } \\
\text { tion and } \\
\text { it is not } \\
\text { done in } \\
\text { Nigeria } \\
\end{array}$ \\
\hline 5 & $\begin{array}{l}\text { Jayathila } \\
\text { ka \& } \\
\text { Sachitra } \\
(2020)\end{array}$ & $\begin{array}{l}\text { Examine the } \\
\text { Drivers to } \\
\text { Engage in } \\
\text { Hybrid } \\
\text { Entre } \\
\text { preneurship } \\
\text { in Sri Lanka }\end{array}$ & $\begin{array}{l}\text { Quant. } \\
\text { research } \\
\text { approach, } \\
\text { primary } \\
\text { data and } \\
\text { binary } \\
\text { logistic } \\
\text { regressio } \\
\text { n }\end{array}$ & $\begin{array}{l}\text { Sufficient } \\
\text { skills } \\
\text { acquired } \\
\text { and } \\
\text { receiving } \\
\text { family } \\
\text { support } \\
\text { have } \\
\text { statistically } \\
\text { significant } \\
\text { influence } \\
\text { for } \\
\text { engaging in } \\
\text { hybrid } \\
\text { entre } \\
\text { preneurship }\end{array}$ & $\begin{array}{l}\text { Poverty } \\
\text { reduc } \\
\text { tion is } \\
\text { not } \\
\text { capture } \\
\mathrm{d} \text { and it } \\
\text { is not } \\
\text { exa } \\
\text { mined } \\
\text { in } \\
\text { Nigeria }\end{array}$ \\
\hline 6 & $\begin{array}{l}\text { Kurcze } \\
\text { wska } \\
\text { and } \\
\text { Mackie } \\
\text { wicz } \\
(2020)\end{array}$ & $\begin{array}{l}\text { Are jacks- } \\
\text { of-all-trades } \\
\text { successful } \\
\text { entre } \\
\text { preneurs? } \\
\text { Revisiting } \\
\text { Lazear's } \\
\text { theory of }\end{array}$ & $\begin{array}{l}\text { Survey } \\
\text { research } \\
\text { design, } \\
\text { primary } \\
\text { data and } \\
\text { Logit } \\
\text { regressio } \\
\text { n }\end{array}$ & $\begin{array}{l}\text { Individuals } \\
\text { with more } \\
\text { diverse } \\
\text { educational } \\
\text { and prof. } \\
\text { background } \\
\text { tend to } \\
\text { have both }\end{array}$ & $\begin{array}{l}\text { The } \\
\text { study } \\
\text { focuses } \\
\text { on } \\
\text { characte } \\
\mathrm{r} \text { istics } \\
\text { of } \\
\text { hybrid }\end{array}$ \\
\hline
\end{tabular}

Hybrid Entrepreneurship as a Tool for Poverty Reduction in Nigeria: A Review of Literature with Qualitative Research and Content Analysis

Page |44| Emerging Markets Journal 


\begin{tabular}{|c|c|c|c|c|c|}
\hline & & $\begin{array}{l}\text { entre } \\
\text { preneurship }\end{array}$ & & $\begin{array}{l}\text { greater } \\
\text { chances of } \\
\text { starting a } \\
\text { company } \\
\text { and being } \\
\text { successful } \\
\text { as hybrid } \\
\text { entre. } \\
\text { compared } \\
\text { to } \\
\text { managerial } \\
\text { experience }\end{array}$ & $\begin{array}{l}\text { entre. } \\
\text { without } \\
\text { conside } \\
\mathrm{r} \text { ing } \\
\text { how it } \\
\text { can } \\
\text { influenc } \\
\mathrm{e} \\
\text { poverty } \\
\text { level in } \\
\text { Nigeria }\end{array}$ \\
\hline 7 & $\begin{array}{l}\text { Iram, } \\
\text { Bilal \& } \\
\text { Rasool } \\
(2020)\end{array}$ & $\begin{array}{l}\text { Hybrid } \\
\text { Entre } \\
\text { preneurship } \\
\text { escalating in } \\
\text { women } \\
\text { academi } \\
\text { cians }\end{array}$ & $\begin{array}{l}\text { Qual. } \\
\text { approach, } \\
\text { snowball } \\
\text { sampling } \\
\text { technique } \\
\text {, primary } \\
\text { data via } \\
\text { interview } \\
\text { and } \\
\text { thematic } \\
\text { analysis }\end{array}$ & $\begin{array}{l}\text { Most } \\
\text { teachers are } \\
\text { facing } \\
\text { financial } \\
\text { constraints } \\
\text { due to low } \\
\text { salary } \\
\text { packages, } \\
\text { single } \\
\text { parenting } \\
\text { and time } \\
\text { flexibility } \\
\text { towards } \\
\text { hybrid } \\
\text { entre } \\
\text { preneurship }\end{array}$ & $\begin{array}{l}\text { The } \\
\text { study } \\
\text { does not } \\
\text { capture } \\
\text { how } \\
\text { hybrid } \\
\text { entre } \\
\text { preneur } \\
\text { ship can } \\
\text { be used } \\
\text { to } \\
\text { reduce } \\
\text { poverty. } \\
\text { Also, it } \\
\text { is not } \\
\text { conduc } \\
\text { ted in } \\
\text { Nigeria } \\
\end{array}$ \\
\hline 8 & $\begin{array}{l}\text { Luc et. } \\
\text { al. } \\
(2018)\end{array}$ & $\begin{array}{l}\text { Hybrid entre } \\
\text { preneurship: } \\
\text { Employees } \\
\text { climbing the } \\
\text { entre } \\
\text { preneurial } \\
\text { ladder }\end{array}$ & $\begin{array}{l}\text { Descrip } \\
\text { tive } \\
\text { research } \\
\text { design, } \\
\text { simple } \\
\text { sampling, } \\
\text { primary } \\
\text { data and } \\
\text { descrip } \\
\text { tive } \\
\text { statistics }\end{array}$ & $\begin{array}{l}\text { Employees' } \\
\text { progress on } \\
\text { the entre } \\
\text { preneurial } \\
\text { ladder is } \\
\text { stimulated } \\
\text { by soft } \\
\text { support and } \\
\text { high wage } \\
\text { job }\end{array}$ & $\begin{array}{l}\text { Poverty } \\
\text { reductio } \\
\mathrm{n} \text { is not } \\
\text { capture } \\
\mathrm{d} \text { in the } \\
\text { paper } \\
\text { and it is } \\
\text { not } \\
\text { conduc } \\
\text { ted in } \\
\text { Nigeria }\end{array}$ \\
\hline 9 & $\begin{array}{l}\text { Indudew } \\
\text { i (2015) }\end{array}$ & $\begin{array}{l}\text { Success } \\
\text { factors of } \\
\text { hybrid entre } \\
\text { preneurs: } \\
\text { Case study } \\
\text { of } \\
\text { Universitas } \\
\text { Ciputra } \\
\text { academician }\end{array}$ & $\begin{array}{l}\text { Qual. } \\
\text { research } \\
\text { approach, } \\
\text { case } \\
\text { study, } \\
\text { expl. } \\
\text { research } \\
\text { design, } \\
\text { and } \\
\text { content } \\
\text { analysis }\end{array}$ & $\begin{array}{l}\text { It is } \\
\text { revealed } \\
\text { that all } \\
\text { academi } \\
\text { cians need } \\
\text { their } \\
\text { lecturing } \\
\text { job to be } \\
\text { successful } \\
\text { as hybrid } \\
\text { entre } \\
\text { preneurs as } \\
\text { well as } \\
\text { good } \\
\text { communi } \\
\text { cation } \\
\text { skills }\end{array}$ & $\begin{array}{l}\text { The } \\
\text { study } \\
\text { does not } \\
\text { focus } \\
\text { on } \\
\text { poverty } \\
\text { reduc } \\
\text { tion and } \\
\text { it is not } \\
\text { done in } \\
\text { Nigeria }\end{array}$ \\
\hline 10 & $\begin{array}{l}\text { Schulz, } \\
\text { Urbig, } \\
\& \\
\text { Procher, } \\
(2017)\end{array}$ & $\begin{array}{l}\text { The role of } \\
\text { hybrid } \\
\text { entrepreneur } \\
\text { ship in } \\
\text { explaining } \\
\text { multiple job } \\
\text { holders' } \\
\text { earnings } \\
\text { structure }\end{array}$ & $\begin{array}{l}\text { Sec. data } \\
\text { from } \\
\text { British } \\
\text { Househld } \\
\text {. Panel } \\
\text { Survey } \\
\text { and logit } \\
\text { regressio } \\
\text { n }\end{array}$ & $\begin{array}{l}\text { Self- } \\
\text { employ } \\
\text { ment is } \\
\text { more } \\
\text { profitable } \\
\text { to earnings } \\
\text { than paid } \\
\text { employ } \\
\text { ment }\end{array}$ & $\begin{array}{l}\text { The } \\
\text { study is } \\
\text { not } \\
\text { done in } \\
\text { Nigeria } \\
\text { and } \\
\text { poverty } \\
\text { reduc } \\
\text { tion is } \\
\text { not } \\
\text { capture } \\
\text { d } \\
\end{array}$ \\
\hline 11 & $\begin{array}{l}\text { Folta, } \\
\text { Delmar } \\
\text { and } \\
\text { Wennbe } \\
\text { rg } \\
(2010)\end{array}$ & $\begin{array}{l}\text { Hybrid } \\
\text { entrepreneur } \\
\text { ship in } \\
\text { Sweden }\end{array}$ & $\begin{array}{l}\text { Primary } \\
\text { data, } \\
\text { multi- } \\
\text { stage } \\
\text { sampling } \\
\text { and } \\
\text { EPRO } \\
\text { (Entre } \\
\text { preneuria } \\
1 \\
\text { Processes } \\
\text { Database) } \\
\end{array}$ & $\begin{array}{l}\text { Hybrid } \\
\text { entry } \\
\text { influences } \\
\text { self-employ } \\
\text { ment }\end{array}$ & $\begin{array}{l}\text { The } \\
\text { study is } \\
\text { not } \\
\text { done in } \\
\text { Nigeria } \\
\text { and } \\
\text { poverty } \\
\text { reduc } \\
\text { tion is } \\
\text { not } \\
\text { capture } \\
\text { d }\end{array}$ \\
\hline 12 & $\begin{array}{l}\mathrm{Xi}, \\
\text { Block, } \\
\text { Lasch, }\end{array}$ & $\begin{array}{l}\text { Mode of } \\
\text { entry into } \\
\text { hybrid }\end{array}$ & $\begin{array}{l}\text { Syste } \\
\text { matic } \\
\text { sampling } \\
\end{array}$ & $\begin{array}{l}\text { It is } \\
\text { indicated } \\
\text { that }\end{array}$ & $\begin{array}{l}\text { Poverty } \\
\text { reduc } \\
\text { tion is }\end{array}$ \\
\hline
\end{tabular}

\begin{tabular}{|c|c|c|c|c|c|}
\hline & $\begin{array}{l}\text { Robert } \\
\text { and } \\
\text { Thurik } \\
(2017)\end{array}$ & $\begin{array}{l}\text { entrepreneur } \\
\text { ship: New } \\
\text { venture } \\
\text { start-up } \\
\text { versus } \\
\text { business } \\
\text { takeover }\end{array}$ & $\begin{array}{l}\text { method, } \\
\text { primary } \\
\text { data and } \\
\text { regressio } \\
\mathrm{n} \text { analysis }\end{array}$ & $\begin{array}{l}\text { educational } \\
\text { attainment } \\
\text { and } \\
\text { manage } \\
\text { ment } \\
\text { experience } \\
\text { are } \\
\text { associated } \\
\text { with new } \\
\text { venture } \\
\text { start-up }\end{array}$ & $\begin{array}{l}\text { not } \\
\text { capture } \\
\mathrm{d} \text { in the } \\
\text { paper } \\
\text { and it is } \\
\text { not } \\
\text { conduc } \\
\text { ted in } \\
\text { Nigeria }\end{array}$ \\
\hline 13 & $\begin{array}{l}\text { Hussaini } \\
\& \\
\text { Ibrahim } \\
(2019)\end{array}$ & $\begin{array}{l}\text { Entrepreneu } \\
\mathrm{r} \text { ship as a } \\
\text { viable tool } \\
\text { for poverty } \\
\text { reduction in } \\
\text { Nigeria }\end{array}$ & $\begin{array}{l}\text { Quant. } \\
\text { research } \\
\text { approach, } \\
\text { primary } \\
\text { data, } \\
\text { syste } \\
\text { matic } \\
\text { sampling } \\
\text { and } \\
\text { partial } \\
\text { least } \\
\text { square }\end{array}$ & $\begin{array}{l}\text { Only entre. } \\
\text { education } \\
\text { and } \\
\text { attraction } \\
\text { are } \\
\text { significantl } \\
\text { y related to } \\
\text { poverty } \\
\text { reduction. } \\
\text { Intention } \\
\text { and } \\
\text { knowledge } \\
\text { are not } \\
\text { related to } \\
\text { poverty } \\
\text { reduction }\end{array}$ & $\begin{array}{l}\text { Despite } \\
\text { being } \\
\text { done in } \\
\text { Nigeria, } \\
\text { the } \\
\text { study } \\
\text { does not } \\
\text { capture } \\
\text { hybrid } \\
\text { entrepre } \\
\text { neurshi } \\
\text { p }\end{array}$ \\
\hline 14 & $\begin{array}{l}\text { Ikechuk } \\
\text { wu, } \\
\text { Onyi } \\
\text { and } \\
\text { Edeh } \\
(2017)\end{array}$ & $\begin{array}{l}\text { Entrepre } \\
\text { neurship } \\
\text { and Poverty } \\
\text { Reduction } \\
\text { in Enugu } \\
\text { State, } \\
\text { Nigeria }\end{array}$ & $\begin{array}{l}\text { Survey } \\
\text { research } \\
\text { design, } \\
\text { primary } \\
\text { data, and } \\
\text { inferentia } \\
\text { 1 statistics }\end{array}$ & $\begin{array}{l}\text { There is a } \\
\text { positive } \\
\text { and } \\
\text { significant } \\
\text { relationship } \\
\text { between } \\
\text { entrepre } \\
\text { neurship } \\
\text { and poverty } \\
\text { reduction }\end{array}$ & $\begin{array}{l}\text { The } \\
\text { study is } \\
\text { conduc } \\
\text { ted in a } \\
\text { state of } \\
\text { Nigeria. } \\
\text { The } \\
\text { study } \\
\text { does not } \\
\text { capture } \\
\text { hybrid } \\
\text { entre } \\
\text { preneur } \\
\text { ship }\end{array}$ \\
\hline 15 & $\begin{array}{l}\text { Ezeanye } \\
\text { ji et al } \\
(2019)\end{array}$ & $\begin{array}{l}\text { Entre } \\
\text { preneurship } \\
\text { developmen } \\
\mathrm{t} \text { and } \\
\text { poverty } \\
\text { reduction in } \\
\text { Nigeria }\end{array}$ & $\begin{array}{l}\text { Qual. } \\
\text { research } \\
\text { approach, } \\
\text { narrative- } \\
\text { textual } \\
\text { case } \\
\text { study } \\
\text { (NTCS) } \\
\text { and } \\
\text { content } \\
\text { analysis }\end{array}$ & $\begin{array}{l}\text { The study } \\
\text { shows that } \\
\text { the state of } \\
\text { depravation } \\
\text { can only be } \\
\text { remedied } \\
\text { through the } \\
\text { develop } \\
\text { ment of } \\
\text { entrepreneu } \\
\text { rship skills } \\
\text { among the } \\
\text { citizens }\end{array}$ & $\begin{array}{l}\text { Despite } \\
\text { being } \\
\text { done in } \\
\text { Nigeria, } \\
\text { the } \\
\text { study } \\
\text { does not } \\
\text { capture } \\
\text { hybrid } \\
\text { entre } \\
\text { preneur } \\
\text { ship }\end{array}$ \\
\hline 16 & $\begin{array}{l}\text { Ibitoye, } \\
\text { Atoyebi } \\
\text { and } \\
\text { Sufian } \\
(2015)\end{array}$ & $\begin{array}{l}\text { Entrepreneu } \\
\mathrm{r} \text { ship and } \\
\text { poverty } \\
\text { reduction in } \\
\text { Nigeria }\end{array}$ & $\begin{array}{l}\text { Stratified } \\
\text { random } \\
\text { sampling, } \\
\text { primary } \\
\text { data and } \\
\text { correlatio } \\
\text { n analysis }\end{array}$ & $\begin{array}{l}\text { There } \\
\text { exists a } \\
\text { positive } \\
\text { and } \\
\text { significant } \\
\text { relationship } \\
\text { between } \\
\text { entrepreneu } \\
\text { rship and } \\
\text { poverty } \\
\text { reduction }\end{array}$ & $\begin{array}{l}\text { The } \\
\text { study is } \\
\text { not } \\
\text { extende } \\
\text { d to } \\
\text { hybrid } \\
\text { entre } \\
\text { preneur } \\
\text { ship }\end{array}$ \\
\hline 17 & $\begin{array}{l}\text { Cannon } \\
\text { (2019) }\end{array}$ & $\begin{array}{l}\text { Hybrid } \\
\text { entre.s' } \\
\text { intention to } \\
\text { transition to } \\
\text { full } \\
\text { entrepreneur } \\
\text { ship: A } \\
\text { career } \\
\text { approach }\end{array}$ & $\begin{array}{l}\text { Survey } \\
\text { research } \\
\text { design, } \\
\text { primary } \\
\text { data and } \\
\text { regressio } \\
\text { n analysis }\end{array}$ & $\begin{array}{l}\text { The study } \\
\text { informs } \\
\text { that hybrid } \\
\text { entrepreneu } \\
\text { rship is a } \\
\text { start-up to } \\
\text { career } \\
\text { develop } \\
\text { ment into } \\
\text { transitionin } \\
\text { g to full } \\
\text { entrepre } \\
\text { neurship }\end{array}$ & $\begin{array}{l}\text { The } \\
\text { study is } \\
\text { not } \\
\text { done in } \\
\text { Nigeria } \\
\text { and } \\
\text { poverty } \\
\text { reduc } \\
\text { tion is } \\
\text { not } \\
\text { capture } \\
\text { d }\end{array}$ \\
\hline 18 & $\begin{array}{l}\text { Hammar } \\
\text { stro \& } \\
\text { Nylen } \\
(2014)\end{array}$ & $\begin{array}{l}\text { Pursuing } \\
\text { hybrid entre } \\
\text { preneurship } \\
\text { in academia }\end{array}$ & $\begin{array}{l}\text { Qual. } \\
\text { research } \\
\text { approach } \\
\text { and } \\
\text { content } \\
\text { analysis }\end{array}$ & $\begin{array}{l}\text { The study } \\
\text { reveals that } \\
\text { hybrid } \\
\text { entre } \\
\text { preneurship } \\
\text { is a good } \\
\text { method for } \\
\text { someone }\end{array}$ & $\begin{array}{l}\text { The } \\
\text { study } \\
\text { exclude } \\
\text { d } \\
\text { poverty } \\
\text { reduc } \\
\text { tion. } \\
\text { Also, it }\end{array}$ \\
\hline
\end{tabular}




\begin{tabular}{|c|c|c|c|c|c|}
\hline & & & & $\begin{array}{l}\text { who wishes } \\
\text { to lower } \\
\text { their } \\
\text { financial } \\
\text { risks when } \\
\text { developing } \\
\text { their own } \\
\text { business }\end{array}$ & $\begin{array}{l}\text { is done } \\
\text { in } \\
\text { Nigeria }\end{array}$ \\
\hline 19 & $\begin{array}{l}\text { Ferreira, } \\
\text { Ferguso } \\
\text { n \& Pitt } \\
(2019)\end{array}$ & $\begin{array}{l}\text { Entre } \\
\text { preneurial } \\
\text { marketing } \\
\text { and hybrid } \\
\text { entrepreneur } \\
\text { ship: The } \\
\text { case of JM } \\
\text { Reid } \\
\text { Bamboo } \\
\text { Rods }\end{array}$ & $\begin{array}{l}\text { Case } \\
\text { study } \\
\text { research } \\
\text { design } \\
\text { primary } \\
\text { data via } \\
\text { obser } \\
\text { vation } \\
\text { and } \\
\text { content } \\
\text { analysis }\end{array}$ & $\begin{array}{l}\text { Entre } \\
\text { preneurial } \\
\text { marketing } \\
\text { enhances } \\
\text { the success } \\
\text { of an } \\
\text { individual } \\
\text { hybrid } \\
\text { entrepre } \\
\text { neurship }\end{array}$ & $\begin{array}{l}\text { Poverty } \\
\text { reduc } \\
\text { tion is } \\
\text { not } \\
\text { conside } \\
\text { red in } \\
\text { the } \\
\text { study } \\
\text { and the } \\
\text { study is } \\
\text { not } \\
\text { exami } \\
\text { ned in } \\
\text { Nigeria }\end{array}$ \\
\hline 20 & $\begin{array}{l}\text { Solesvik } \\
(2017)\end{array}$ & $\begin{array}{l}\text { Hybrid } \\
\text { entrepre } \\
\text { neurship: } \\
\text { How and } \\
\text { Why } \\
\text { Entrepre } \\
\text { neurs } \\
\text { Combine } \\
\text { Employmen } \\
\text { t with Self- } \\
\text { Employmen } \\
\mathrm{t}\end{array}$ & $\begin{array}{l}\text { Case } \\
\text { study } \\
\text { research } \\
\text { design } \\
\text { and } \\
\text { content } \\
\text { analysis }\end{array}$ & $\begin{array}{l}\text { Hybrid } \\
\text { entre } \\
\text { preneurship } \\
\text { is a viable } \\
\text { path for } \\
\text { people } \\
\text { willing to } \\
\text { realize } \\
\text { their entre } \\
\text { preneurial } \\
\text { ambitions } \\
\text { in a lower- } \\
\text { risk and } \\
\text { organic } \\
\text { way }\end{array}$ & $\begin{array}{l}\text { The } \\
\text { study is } \\
\text { not } \\
\text { done in } \\
\text { Nigeria } \\
\text { and } \\
\text { poverty } \\
\text { reduc } \\
\text { tion is } \\
\text { not } \\
\text { capture } \\
\text { d }\end{array}$ \\
\hline 21 & $\begin{array}{l}\text { Kritskay } \\
\text { a, } \\
\text { Kolverei } \\
\text { d and } \\
\text { Isaksen } \\
\text { (2017) }\end{array}$ & $\begin{array}{l}\text { Hybrid entre } \\
\text { preneurs: } \\
\text { Character } \\
\text { istics and } \\
\text { achieve } \\
\text { ments }\end{array}$ & $\begin{array}{l}\text { Descrip } \\
\text { tive } \\
\text { research } \\
\text { design, } \\
\text { primary } \\
\text { data via } \\
\text { question } \\
\text { naire and } \\
\text { descrip } \\
\text { tive } \\
\text { statistics }\end{array}$ & $\begin{array}{l}\text { The study } \\
\text { indicates } \\
\text { that most of } \\
\text { the new } \\
\text { businesses } \\
\text { are started } \\
\text { by hybrid }\end{array}$ & $\begin{array}{l}\text { The } \\
\text { study is } \\
\text { not } \\
\text { done in } \\
\text { Nigeria } \\
\text { and } \\
\text { poverty } \\
\text { reduc } \\
\text { tion is } \\
\text { not } \\
\text { capture } \\
\text { d }\end{array}$ \\
\hline 22 & $\begin{array}{l}\text { Dzomon } \\
\text { da \& } \\
\text { Fatoki } \\
(2018)\end{array}$ & $\begin{array}{l}\text { Demystify } \\
\text { ing the } \\
\text { motivations } \\
\text { towards } \\
\text { hybrid entre } \\
\text { preneurship } \\
\text { among the } \\
\text { working } \\
\text { populace in } \\
\text { South } \\
\text { Africa }\end{array}$ & $\begin{array}{l}\text { Quant. } \\
\text { research, } \\
\text { conven } \\
\text { ient } \\
\text { sampling } \\
\text { method, } \\
\text { primary } \\
\text { data and } \\
\text { t-tests }\end{array}$ & $\begin{array}{l}\text { There is no } \\
\text { significant } \\
\text { gender } \\
\text { difference } \\
\text { on hybrid } \\
\text { entrepre } \\
\text { neurship. It } \\
\text { further } \\
\text { showed } \\
\text { that } \\
\text { participants } \\
\text { are } \\
\text { motivated } \\
\text { mainly by } \\
\text { both a } \\
\text { desire to } \\
\text { supplement } \\
\text { income and } \\
\text { derive non- } \\
\text { monetary } \\
\text { benefits }\end{array}$ & $\begin{array}{l}\text { The } \\
\text { study } \\
\text { fails to } \\
\text { capture } \\
\text { poverty } \\
\text { reduc } \\
\text { tion. } \\
\text { Also, it } \\
\text { is not } \\
\text { carried } \\
\text { out in } \\
\text { Nigeria }\end{array}$ \\
\hline 23 & $\begin{array}{l}\text { Petrova } \\
\text { (2012) }\end{array}$ & $\begin{array}{l}\text { Entrepre } \\
\text { neurial } \\
\text { dynamics } \\
\text { and hybrid } \\
\text { entrepreneur } \\
\text { ship }\end{array}$ & $\begin{array}{l}\text { Primary } \\
\text { data, } \\
\text { descrip } \\
\text { tive } \\
\text { statistics } \\
\text { and } \\
\text { inferentia } \\
\text { 1 statistics }\end{array}$ & $\begin{array}{l}\text { Financial } \\
\text { constraints } \\
\text { do not } \\
\text { impact } \\
\text { hybrid } \\
\text { entrepre } \\
\text { neurship. } \\
\text { Whilst, } \\
\text { changes in } \\
\text { the labor } \\
\text { market may } \\
\text { be driving }\end{array}$ & $\begin{array}{l}\text { The } \\
\text { study } \\
\text { fails to } \\
\text { capture } \\
\text { poverty } \\
\text { reductio } \\
\text { n. Also, } \\
\text { it is not } \\
\text { carried } \\
\text { out in } \\
\text { Nigeria }\end{array}$ \\
\hline
\end{tabular}

\begin{tabular}{|c|c|c|c|c|c|}
\hline & & & & $\begin{array}{l}\text { individuals } \\
\text { towards } \\
\text { hybrid } \\
\text { entre } \\
\text { preneurship }\end{array}$ & \\
\hline 24 & $\begin{array}{l}\text { Raffiee } \\
\text { and } \\
\text { Feng } \\
(2014)\end{array}$ & $\begin{array}{l}\text { Small-scale } \\
\text { entry via } \\
\text { hybrid entre } \\
\text { preneurship } \\
\text { as a real } \\
\text { option }\end{array}$ & $\begin{array}{l}\text { Qual. } \\
\text { research } \\
\text { approach } \\
\text { and } \\
\text { descrip } \\
\text { tive } \\
\text { statistics }\end{array}$ & $\begin{array}{l}\text { Hybrid } \\
\text { entre } \\
\text { preneurs } \\
\text { could } \\
\text { choose to } \\
\text { either } \\
\text { invest } \\
\text { heavily in } \\
\text { their entre } \\
\text { preneurial } \\
\text { project or } \\
\text { exit easily } \\
\text { from it, } \\
\text { depending } \\
\text { upon the } \\
\text { early } \\
\text { returns }\end{array}$ & $\begin{array}{l}\text { The } \\
\text { study } \\
\text { fails to } \\
\text { capture } \\
\text { poverty } \\
\text { reduc } \\
\text { tion. } \\
\text { Also, it } \\
\text { is not } \\
\text { carried } \\
\text { out in } \\
\text { Nigeria }\end{array}$ \\
\hline 25 & $\begin{array}{l}\text { Viljama, } \\
\& \\
\text { EVaram } \\
\text { äki } \\
(2015)\end{array}$ & $\begin{array}{l}\text { Do } \\
\text { Persistent } \\
\text { and } \\
\text { Transitory } \\
\text { Hybrid } \\
\text { Entrepreneu } \\
\text { rs Differ? }\end{array}$ & $\begin{array}{l}\text { Survey } \\
\text { research } \\
\text { design, } \\
\text { primary } \\
\text { data via } \\
\text { question } \\
\text { naire and } \\
\text { descrip } \\
\text { tive } \\
\text { statistics }\end{array}$ & $\begin{array}{l}\text { It is } \\
\text { indicated } \\
\text { that not all } \\
\text { hybrid } \\
\text { entre } \\
\text { preneurs } \\
\text { are } \\
\text { intended to } \\
\text { transit to } \\
\text { full entre } \\
\text { preneurship }\end{array}$ & $\begin{array}{l}\text { The } \\
\text { study } \\
\text { fails to } \\
\text { capture } \\
\text { poverty } \\
\text { reduc } \\
\text { tion. } \\
\text { Also, t } \\
\text { is not } \\
\text { carried } \\
\text { out in } \\
\text { Nigeria }\end{array}$ \\
\hline 26 & $\begin{array}{l}\text { Schulz, } \\
\text { Urbig \& } \\
\text { Procher } \\
(2016)\end{array}$ & $\begin{array}{l}\text { Hybrid entre } \\
\text { preneurship } \\
\text { and public } \\
\text { policy: The } \\
\text { case of firm } \\
\text { entry } \\
\text { deregulation }\end{array}$ & $\begin{array}{l}\text { Survey } \\
\text { research } \\
\text { design, } \\
\text { primary } \\
\text { data via } \\
\text { question } \\
\text { naire and } \\
\text { logit } \\
\text { regressio } \\
\text { n }\end{array}$ & $\begin{array}{l}\text { Hybrid } \\
\text { entre } \\
\text { preneurs } \\
\text { are more } \\
\text { responsive } \\
\text { to changes } \\
\text { in entry } \\
\text { regulation } \\
\text { than full- } \\
\text { time } \\
\text { entrepre } \\
\text { neurs. } \\
\text { However, } \\
\text { educated } \\
\text { hybrid } \\
\text { entrepre } \\
\text { neurs } \\
\text { respond to } \\
\text { changes } \\
\text { more than } \\
\text { the } \\
\text { uneducated }\end{array}$ & $\begin{array}{l}\text { The } \\
\text { study } \\
\text { fails to } \\
\text { capture } \\
\text { poverty } \\
\text { reduc } \\
\text { tion. } \\
\text { Also, it } \\
\text { is not } \\
\text { carried } \\
\text { out in } \\
\text { Nigeria }\end{array}$ \\
\hline 27 & $\begin{array}{l}\text { Cestino } \\
(2019)\end{array}$ & $\begin{array}{l}\text { Hybrid entre } \\
\text { preneurship } \\
\text { as the } \\
\text { pursuit of } \\
\text { valued } \\
\text { forms of } \\
\text { work }\end{array}$ & $\begin{array}{l}\text { Qual. } \\
\text { research } \\
\text { design, } \\
\text { multi } \\
\text { case- } \\
\text { study } \\
\text { research } \\
\text { design } \\
\text { and } \\
\text { thematic } \\
\text { analysis }\end{array}$ & $\begin{array}{l}\text { It is } \\
\text { revealed } \\
\text { that both } \\
\text { monetary } \\
\text { and non- } \\
\text { monetary } \\
\text { benefits are } \\
\text { the factors } \\
\text { that } \\
\text { enhance } \\
\text { hybrid } \\
\text { entre } \\
\text { preneurship }\end{array}$ & $\begin{array}{l}\text { The } \\
\text { study } \\
\text { fails to } \\
\text { capture } \\
\text { poverty } \\
\text { reduc } \\
\text { tion. } \\
\text { Also, it } \\
\text { is not } \\
\text { carried } \\
\text { out in } \\
\text { Nigeria }\end{array}$ \\
\hline
\end{tabular}

Source: Authors' own compilation

\section{Findings and Discussion}

One of the contemporary issues in entrepreneurship is hybrid entrepreneurship. Hybrid entrepreneurship is very important for the survival of individuals, especially in developing economies like Nigeria. The challenges in Nigerian economy have made living conditions for Nigerians difficult to bear. The high rate of inflation as a 
result of high prices of goods and services, high rent and high transportation costs among other have weakened the purchasing power of Nigerians. The findings show that, hybrid entrepreneurship helps individual to comfortably meet their daily expenses. It is a source of additional income, but not yet popular in Nigeria.

Additionally, it is observed that there are studies on hybrid entrepreneurship in the globe. Most of the studies are not done in Africa, especially in Nigeria as they are conducted in developed countries. The study of Aladejebi (2020) on hybrid entrepreneurship does not capture poverty reduction. Relatively, the studies of Hussaini and Ibrahim (2019), Ezeanyeji et. al. (2019), Ikechukwu, Onyi and Edeh (2017), Ibitoye, Atoyebi and Sufian (2015) examine entrepreneurship and poverty reduction in Nigeria, but hybrid entrepreneurship is not captured. This is a strong evidence that, more about hybrid entrepreneurship need to be examined.

It is obvious that much focus has not been given in the literature to how hybrid entrepreneurship could reduce poverty level, because most of the studies are from developed economies where poverty is not a major issue of concern. The few studies in Nigeria and probably in developing economies are only looking for how entrepreneurship activities could be promoted. What is prevalent in Nigeria and many other developing economies is part-time entrepreneurship which has not enhanced poverty reduction as such, because there is still evidence of poverty from the contextual observation. This could be as a result of the legal framework as it operates in Nigeria. Besides, the medical and legal professionals running self-employment with official jobs are not tolerated as such. Government and many private organizations see this as divided interest, which will jeopardize the main interest of organization. Also, it could be as a result of poor risk taking ability and poor income that are not sufficient to engage in extra activity.

\section{Conclusion}

Literature shows that, hybrid entrepreneurship has elevated some notable businessmen and businesswomen to achieve their personal goals for becoming billionaires of the world. For instance, Steve Wozniak as the founder of Apple worked in a paid job while running his personal business. Similarly, Pierre Omidyar was a paid personnel at a software development firm when he founded e-bay.

Thus, one could see that hybrid entrepreneurship will lift individuals out of poverty to the point of becoming millionaires if embraced. It is concluded that, hybrid entrepreneurship is a determinant practice to reduce the rate of poverty in Nigeria because of availability of many untapped resources and opportunity. Thus, hybrid entrepreneurship has several advantages in terms of economics and finance.

Therefore, the study recommends that hybrid entrepreneurship should be encouraged among the citizens in Nigeria to reduce the poverty level. Also, every Nigerian should try to get involved or engage in other businesses to augment their income for achieving their personal and family goals. This would create jobs and minimize the poverty level.

\section{References}

Astebro, T., \& Thompson, P. (2011). Entrepreneurs, jacks of all trades or hobos? Research Policy, 40(5), 637-649.

Aladejebi, O. (2020). Hybrid entrepreneurship: Combining employment and self-employment in Nigeria. Archives of Business Research, 8(7). 404-418.

Alvarez, S.A., \& Barney, J.B. (2014). Entrepreneurial opportunities and poverty alleviation. Entrepreneurship: Theory and Practice, 38(1), 159-184.

Backes-Gellner, U., \& Moog, P. (2013). The disposition to become an entrepreneur and the jacks-of-alltrades in social and human capital. The Journal of Socio-Economics, 47, 55-72.

Bögenhold, D. (2019). From hybrid entrepreneurs to entrepreneurial billionaires: Observations on the socioeconomic heterogeneity of selfemployment. American Behavioral Scientist, 63(2), 129-146.

Cannon, S. (2019). Hybrid entrepreneurs' intention to transition to full entrepreneurship: A career approach. Ph.D Dissertation, Georgia State University.

Cestino, J. (2019). Hybrid entrepreneurship as the pursuit of valued forms of work. Academy of Management, 1, 1-13.

Clark, D. (2018). How to get your side hustle off the ground. Harvard Business Review.

Demir, C., Werner, A., Kraus, S., \& Jones, P. (2020). Hybrid entrepreneurship: A systematic literature review. Journal of Small Business \& Entrepreneurship, 1, 82-91.

Dzomonda, O., \& Fatoki, O. (2018). Demystifying the motivations towards hybrid entrepreneurship among the working populace in South Africa. Academy of Entrepreneurship Journal, 24(4), $1-9$.

Ezeanyeji, C.I., Imoagwu, C.P., \& Ejefobihi, U.F. (2019). Entrepreneurship development and poverty reduction in Nigeria: The synergy. International Journal of Economics, Commerce and Management, 7(4), 584-601.

Ferreira, C.C. (2020). Experiential learning theory and hybrid entrepreneurship: factors influencing the transition to full-time entrepreneurship. International Journal of Entrepreneurial Behavior \& Research, 26(8), 1845-1863. 
Ferreira, C., Ferguson, S.L., \& Pitt, L.F. (2019). Entrepreneurial marketing and hybrid entrepreneurship: the case of JM Reid Bamboo Rods. Journal of Marketing Management, 35(9-10), 867-885.

Florida, R. (2014). The rise of the creative class, revisited. New York City, NY: Basic Books.

Folta, T.B., Delmar, F., \& Wennberg, K. (2010). Hybrid entrepreneurship. Management Science, 56(2), 253-269.

Hammarström, N., \& Nylén, V. (2014). Pursuing hybrid entrepreneurship in Academia. Retrieved from $\mathrm{http} / / / \mathrm{www}$.diva-

portal.org/smash/get/diva2:730441/FULLTEX T01.pdf

Hartog, J., Van Praag, M., \& Van Der Sluis, J. (2010). If you are so smart, why aren't you an entrepreneur? Returns to cognitive and social ability: Entrepreneurs versus employees. Journal of Economics and Management Strategy, 19(4), 947-989.

Hussaini, U., \& Noma, I.A. (2019). Entrepreneurship as a viable tool for poverty reduction in Nigeria. International Journal of Commerce and Management Research, 5(1), 5-12.

Ibitoye, O., Atoyebi, K. O. \& Sufian. J.B. (2015). Entrepreneurship and poverty reduction in Nigeria: An empirical analysis Journal of Business and Management, 17(3), 16-19.

Ikechukwu, D., Onyi, A. J., \& Edeh, F. O. (). Entrepreneurship and poverty reduction in Nigeria: Empirical analysis of industrial layout Coal Camp, Enugu-State, Nigeria. Journal of Educational Policy and Entrepreneurial Research, 4(3), 115-130.

Indudewi, F.Y.R. (2015). Success factors of hybrid entrepreneurs: Case study of Universitas Ciputra academician. Mediterranean Journal of Social Sciences, 6(5), 84-90.

Iram, T., Bilal, A.R., \& Rasool, Y. (). Hybrid Entrepreneurship escalating in women academicians. Paideuma Journal, 8(8), 86-106.

Jayathilaka, G., \& Sachitra, V. (2020). Examine the drivers to engage in hybrid entrepreneurship in Sri Lanka. Sri Lankan Journal of Entrepreneurship, 2(1), 102-131.

Kelley, D. J., Singer, S., \& Herrington, M. (2016). Global entrepreneurship monitor: 2015/2016 Global Report. London: Global Entrepreneurship Research Association.

Kim, P., Aldrich, H., \& Keister, L. (2006). The impact of financial, human, and cultural capital on entrepreneurial entry in the United States. Small Business Economics, 27, 5-22.
Kuhn, K. (2016). The rise of the gig economy and implications for understanding work and workers. Industrial and Organizational Psychology, 9(1), 157-162.

Kurczewska, A., \& Mackiewicz, M. (2020). Are jacksof-all-trades successful entrepreneurs? Revisiting Lazear's theory of entrepreneurship. Baltic Journal of Management, 1, 1-21.

Kurczewska, A., Mackiewicz, M., Dory, W., \& Wawrzyniak, D. (2020). Peculiarity of hybrid entrepreneurs - revisiting Lazear's theory of entrepreneurship. Journal of Business Economics and Management, 21(1), 277-300.

LKritskaya, L., Kolvereid, L., \& Isaksen, E.J. (2017). Hybrid entrepreneurs: characteristics and achievements. De Boeck Supérieur, 34, 7-19.

Luc, S., Chirita, G., Delvaux, E., \& Kepnou, A. (2018). Hybrid entrepreneurship: Employees climbing the entrepreneurial ladder. International Review of Entrepreneurship, 16(1), 89-113.

Nordström, C. (2015). The passionate combining entrepreneurs. Ph.D Thesis, Mid Sweden University, Sweden.

Petrova, K. (2010). Part-time entrepreneurship, learning and ability. Journal of Management Policy and Practice, 12(1), 64-75.

Petrova, K. (2012). Part-time entrepreneurship and financial constraints: Evidence from the panel study of entrepreneurial dynamics. Small Business Economics, 39(2), 473-493.

Popiel, P. (2017). Boundaryless in the creative economy: Assessing freelancing on upwork. Critical Studies in Media Communication, 34(3), 1-14.

Raffiee, J., \& Feng, J. (2014). Should I quit my day job? A hybrid path to entrepreneurship. Academy of Management Journal, 57(4), 936-963.

Schulz, M., Urbig, D., \& Procher, V. (2016). Hybrid entrepreneurship and public policy: The case of firm entry deregulation. Journal of Business Venturing, 31(3), 272-286.

Schulza, M., Urbiga, D., \& Prochera, V. (2017). The role of hybrid entrepreneurship in explaining multiple job holders' earnings structure. Journal of Business Venturing Insights, 7, 914

Solesvik, M.Z. (2017). Hybrid entrepreneurship: How and why entrepreneurs combine employment with self-employment. Technology Innovation Management Review, 7(3), 33-41.

Stuetzer, M., Obschonka, M., Davidsson, P., \& SchmittRodermund, E. (2013). Where do entrepreneurial skills come from? Applied Economics Letters, 20(12), 1183-1186. 
Tegtmeier, S., Kurczewska, A., \& Halberstadt, J. (2016). Are women graduates jacquelines-of-alltrades? Challenging Lazear's view on entrepreneurship. Small Business Economics, 47(1), 77-94.

Umaru, H., \& Ibrahim, A. (2019). Entrepreneurship as a viable tool for poverty reduction in Nigeria. International Journal of Commerce and Management Research, 5(1), 5-12.

Viljamaa, A., \& Varamäki, E. (2014), Part-time or nascent entrepreneurs? Academic hybrid Entrepreneurship. In: Managing in an interconnected world: Pioneering business and technology excellence, Global Business and Technology Association 16th Annual International Conference Readings Book, July 8th-12th, Baku, Azerbaijan, 636-643.

Viljamaa, A., \& Varamäki, E. (2015). Do persistent and transitory hybrid entrepreneurs differ? International Journal of Economics and Management Engineering, 9(3), 936-940.

Viljamaa, A., Varamäki, E., \& Joensuu-Salo, S. (2017). Best of both worlds? Persistent hybrid entrepreneurship. Journal of Enterprising Culture, 24(4), 56-71.

Viljamaa, A.H., \& EVaramäki, E.M. (2015). Do persistent and transitory hybrid entrepreneurs differ?. International Journal of Economics and Management Engineerin, 9(3), 936-940.

Wagner, J. (2003). Testing Lazear's jack-of-all-trades view of entrepreneurship with German microdata. Applied Economics Letters, 10(11), 687689.

Wagner, J. (2006). Are nascent entrepreneurs 'Jacks-ofall-trades'? A test of Lazear's theory of entrepreneurship with German data. Applied Economics, 38(20) 2415-2419.

World Bank (1990). Nigeria -poverty in the midst of plenty. The challenge of growth inclusive: A world bank poverty assessment. Population and Human Resource, Divisions Report, No. 14733.

World Bank (2011). World development report. New York: Oxford University Press.

Xi, G., Block, J., Lasch, F., Robert, F., \& Thurik, R. (2017). Mode of entry into hybrid entrepreneurship: New venture start-up versus business takeover. IZA Institute of Labor Economics, Discussion Paper Series. 